\title{
ARTICLE
}

\section{A real-time multichannel detector system for large-scale environmental radiation survey}

\author{
Cheng-Chih Liang ${ }^{\mathrm{a}}$, Yen-Fu Chen ${ }^{\mathrm{b}}$, Jeng-Wei Lin ${ }^{\mathrm{a}}$, Chun-Kai Huang ${ }^{\mathrm{a}}$, Wei-Lin Chen ${ }^{\mathrm{a}}$, Rong-Jiun Sheu ${ }^{\mathrm{a}, \mathrm{b}}$, Uei-Tyng \\ Lin $^{\mathrm{c}}$ and Shiang-Huei Jiang ${ }^{\mathrm{a}, \mathrm{b}^{*}}$ \\ ${ }^{a}$ Institute of Nuclear Engineering and Science, National Tsing Hua University, Hsinchu, Taiwan; ${ }^{b}$ Department of Engineering and \\ System Science, National Tsing Hua University, Hsinchu, Taiwan; ${ }^{c}$ Institute of Radiological Sciences, Tzu Chi College of \\ Technology, Hualien, Taiwan
}

\begin{abstract}
In this work, we established a real-time multichannel detector system for large-scale environmental radiation survey. The system consists of a Berkeley Lab cosmic-ray detector for the measurement of muons, a moderated ${ }^{3} \mathrm{He}$ detector for neutrons, a $\mathrm{NaI}(\mathrm{Tl})$ as well as a HPGe spectrometer for terrestrial gamma rays, and a high pressure ionization chamber, which responses to both cosmic rays and terrestrial gamma rays. The measured data of these five detector channels as well as signals from a global position system are acquired and displayed by a LabVIEW program on a notebook computer every one minute (10 min for HPGe and 20 min for $\mathrm{NaI}(\mathrm{Tl})$ gamma-ray spectrometers). The advantage and merit of this multichannel detector system have been demonstrated by test runs with the equipment set up both on a train and on a bus to show in real time the variation of cosmic-ray intensity as a function of altitude and the gamma-ray dose rates as well as the relative concentrations of the natural radioactive elements over a large survey area. This real-time multichannel detector system was originally developed for natural background radiation measurements for popular science education to general public, however, due to the specific features of each detector of the system it appears to be an ideal system for radiation survey over a large radiation polluted or contaminated area resulted from a severe nuclear accident, such as Fukushima Dai-Ichi Nuclear Power Plant accident.
\end{abstract}

\section{Keywords: cosmic-ray muon; cosmic-ray neutron; terrestrial gamma ray; real time measurement; large-} scale measurement; survey measurement; popular science education

\section{Introduction}

Our earth living environment is full of natural background radiation coming from cosmic rays and terrestrial gamma rays as far as external exposure is concerned. Cosmic rays on the ground consist of both directly ionizing components, mainly muons and electrons, and neutrons. The muon flux at sea level in the northern part of the United States is around $1.9 \times 10^{-2}$ $\mathrm{cm}^{-2} \mathrm{sec}^{-1}$. The neutron and electron fluxes are roughly one third and one fourth, respectively, that of muons [1]. The intensity of cosmic rays varies with the altitude roughly in an exponential form [2]. The cosmic ray intensity depends also on the geomagnetic latitude, with which the cosmic-ray neutron intensity increases from $0^{0}$ to $90^{\circ}$ by a factor of around 4 , however, the intensity of the directly ionizing component of cosmic rays has only a mild variation of $\sim 10 \%$. The cosmic ray intensity is even influenced by the 11-year solar cycle: neutron intensity may increase by up to $\sim 25 \%$ and muon intensity by up to $\sim 8 \%$ from solar maximum to solar minimum. The terrestrial gamma rays originating from naturally occurring radioactive elements (U series, Th

*Corresponding author. Email: shjiang@mx.nthu.edu.tw series, and ${ }^{40} \mathrm{~K}$ ) vary widely from one region to another because of variations in the abundance of these elements. In some regions of India and Brazil, where there are extreme areas of thorium-bearing monazite sands, terrestrial gamma ray intensities increase several tens and more than several hundred times, respectively, than the world average level [2].

The aim of this work is to establish a multichannel detector system to carry out large-scale survey measurements of different components of natural background radiation in real time, so as to demonstrate the large intensity variation of natural background radiation in our living environment. The fundamental obstacle of the development and application of nuclear power is, among others, the concern about radioactive waste and radiation contamination. Only when the general public understands radiation correctly, these concerned issues can be confronted and resolved smoothly.

This real-time multichannel detector system was originally developed for natural background radiation measurements for popular science education to general public. Due to the specific features of each detector described at the end of paragraph 2.1, it appears to be an 
ideal system for radiation survey over a large radiation polluted or contaminated area resulted from a severe nuclear accident, such as Fukushima Dai-Ichi Nuclear Power Plant accident.

\section{Materials and methods}

\subsection{Set up of the detector system}

Two types of detectors were selected for the measurement of cosmic rays: a Berkeley Lab cosmic-ray detector was used to measure the directly ionizing components of cosmic rays, mainly muons and a small fraction of high-energy electron, and a moderated ${ }^{3} \mathrm{He}$ proportional counter was applied to measure cosmic-ray neutrons. The Berkeley Lab cosmic-ray detector[3-5] consists of two plastic scintillation counters mounted face to face opposite with a separation distance of $\sim 11$ $\mathrm{cm}$. Each plastic scintillator has an area of $\sim 200 \mathrm{~cm}^{2}$ and a thickness of $0.635 \mathrm{~cm}$. A specially designed circuit board, which contains the functions of high-voltage power supply, preamplifier, amplifier and coincident circuit, is applied to combine the two scintillation counters. Only when a radiation particle passes the two plastic scintillator sheets and produces scintillation signal simultaneously, a signal count will appear through the coincident circuit.

The moderated ${ }^{3} \mathrm{He}$ proportional counter is built from a Reute Stokes high-sensitive ${ }^{3} \mathrm{He}$ proportional counter filled with 4 -atm ${ }^{3} \mathrm{He}$ gas. The ${ }^{3} \mathrm{He}$ proportional counter is coupled with a PDT (Precision Detector Technology, Inc.) module, which is a functional circuit of high-voltage power supply, preamplifier, amplifier, and discriminator, and surrounded by $6.5-\mathrm{cm}$ thick polyethylene to get a maximum counting rate of cosmic-ray neutrons.

For the measurement of terrestrial gamma rays we applied a Reuter Stokes high-pressure ionization chamber (HPIC) to measure the radiation dose rate. The HPIC is a conventional environmental radiation monitor, which is normally installed at site boundaries of nuclear power plants or other radiation emitting facilities. The HPIC is made of a stainless steel sphere with a diameter of $\sim 30 \mathrm{~cm}$ and filled with 20 -atm argon gas. Note that the measured signal of HPIC is caused not only by the terrestrial gamma rays but also by the directly ionizing component of the cosmic rays.

In order to distinguish and identify the origin of the terrestrial gamma rays we originally adopted an ORTEC 3" x 3" NaI(Tl) spectrometer coupled with a digiBase-E base module for the measurement. Four region of interests (ROI) in the measured pulse height spectrum were selected to record gamma rays from different origin: ROI 1 corresponding to gamma rays from ${ }^{214} \mathrm{Bi}$ $(609 \mathrm{keV})$ and ${ }^{208} \mathrm{Tl}(583 \mathrm{keV})$; ROI 2 corresponding to ${ }^{40} \mathrm{~K}(1460 \mathrm{keV})$; ROI 3 corresponding to ${ }^{214} \mathrm{Bi}(1675$ $\mathrm{keV})$; and ROI 4 corresponding to ${ }^{208} \mathrm{Tl}(2620 \mathrm{keV})$. Because the uncertainties of ROI data of measured normal environmental gamma-ray spectra from the 3 " $\mathrm{x}$ 3" $\mathrm{NaI}(\mathrm{Tl})$ spectrometer for an acquisition time of 20 min are large, of the order of more than $10 \%$, later on, we added a CANBERRA GC3520 high purity germanium detector (HPGe) with a counting efficiency of $35 \%$ to the multichannel detector system to supplement or even replace the $\mathrm{NaI}(\mathrm{Tl})$ measurement. For the HPGe measurement the counts of $609 \mathrm{keV}$ $\left({ }^{214} \mathrm{Bi}\right), 1460 \mathrm{keV}\left({ }^{40} \mathrm{~K}\right)$, and $2620 \mathrm{keV}\left({ }^{208} \mathrm{Tl}\right)$ peaks on the pulse height spectrum acquired for $10 \mathrm{~min}$ were taken as an indication of the abundances of ${ }^{238} \mathrm{U},{ }^{40} \mathrm{~K}$, and ${ }^{232} \mathrm{Th}$, respectively, in the earth.

In addition to the five different types of detectors for the measurement of cosmic rays and terrestrial gamma rays, a GARMIN global positioning system (GPS) unit is incorporated in the detector system to record the geographic track of the measurement path. In addition to the longitude and latitude, the altitude data are also provided by the GPS.

All the signals from the five radiation detectors and the GPS are acquired by a LabVIEW program on a notebook computer. Signals from all the devices are acquired every $1 \mathrm{~min}$ except $\mathrm{NaI}(\mathrm{Tl})$ and HPGe detectors, for which the pulse height spectra are acquired every 20 and $10 \mathrm{~min}$, respectively, and the data are stored on the disk and displayed graphically on the screen in real time for visual inspection at any time. In the same time the screen display is sent to the disk of a server in the campus every $1 \mathrm{~min}$ via wireless internet, so that anyone can view the display of the measured data anywhere through the internet. Figure 1 shows the photo of the multichannel detector system. In addition to the survey measurement of the natural background radiation, the established multichannel detector system is also very suitable for radiation survey over a large radiation contaminated area because of the following reasons: The $\mathrm{NaI}(\mathrm{Tl})$ or $\mathrm{HPGe}$ spectrometer bears the capability of distinguishing gamma rays emitted from contaminating radioactive material from those emitted from naturally occurring radioactive elements. The HPIC will respond sensitively to gamma rays emitted from contaminating radioactive material in addition to natural terrestrial gamma rays and ionizing components of cosmic rays. ${ }^{3} \mathrm{He}$ proportional counter and Berkeley Lab cosmic-ray detector won't respond to the contaminating gamma rays and their signals can serve as reference data of the multichannel detector system. Meanwhile, the GPS can provide the geographic data of the contaminated measurement location.

\subsection{Calibration of detectors}

In order to determine the natural background radiation quantitatively, either in dose rate or in activity concentration, the detectors need to be calibrated. Since the emphesis of this work is to carry out real-time large-scale survey measurements, the calibration of some detectors was conducted in an application oriented way. The HPIC was calibrated in terms of exposure rate $(\mu \mathrm{R} / \mathrm{hr})$ at the National Radiation Standard Laboratoty, Taiwan. The Berkeley Lab cosmic ray detector in terms of $\mu \mathrm{R} / \mathrm{hr}$, which is equivalent to air kerma rate, was 


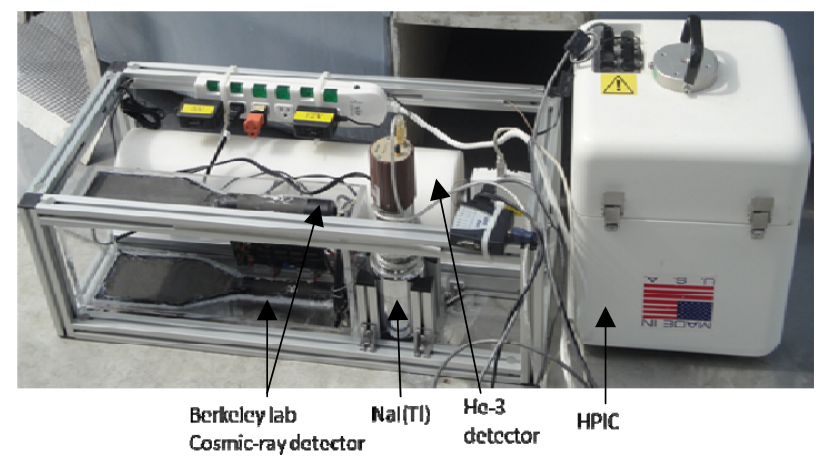

Figure 1. Photo of the multichannel detector system.

made by a measurement performed at the water surface in the middle of Shyr-Men Dan, where the exposure rate measured by the HPIC is essentially contributed only by the directly ionizing cosmic rays. The calibration of the moderated ${ }^{3} \mathrm{He}$ proportional counter was made by measurement on the sea level ground, where the cosmic neutron dose rate has been determined in our previous study [6]. The absolute peak efficiency of the HPGe detector with respective to the earth distributed source or to the ground surface source was determined by the effective solid angle concept proposed by Moens et al. $[7,8]$, according to which the absolute peak efficiency of the HPGe detector to a distributed source can be calculated from the measured reference point-source efficiency multiplied by the ratio of effective solid angle subtended by the detecor over the distributed source to that subtended by the detector at the reference point source.

\section{Results and discussion}

\subsection{Large-scale survey measurement}

For a commissioning test of the established real-time multichannel detector system, the system was set up at two seats on a train to measure the natural background radiation along the railway around the northern Taiwan coast from Hsinchu to Hualien with a distance of $\sim 275$ $\mathrm{km}$ [9]. The measurement was successful. Because the survey was carried out in a tiny region with small variation in altitude and geomagnetic latitude, no significant change in the cosmic-ray muon and neutron counting rates was observed. However, these cosmic-ray counting rates reduced a great deal when the train went underground or in the tunnels. The compositions of the terrestrial gamma rays were found to be essentially unchanged and the variation of the intensity to be mild within a factor of two.

Later on, we mounted the real-time multichannel detector system on a 20 -seat bus and carried out a field survey measurement from Hsinchu to Mount Ho Huan with an altitude of $\sim 3200 \mathrm{~m}$ above sea level in middle Taiwan. Figure 2 shows the measured data display along the way from Hsinchu to Mount Ho Huan and return. Clearly, the cosmic ray intensity increased with the altitude. At Mount Ho Huan, the muon counting rate from the Berkeley cosmic-ray detector increased by a factor of $\sim 2.6$ compared with that in Hsinchu, however, the neutron counting rate from the moderated ${ }^{3} \mathrm{He}$ detector increased by a factor of $\sim 6.5$. Because it is contributed from both cosmic muons and terrestrial gamma rays the dose rate from the HPIC increased only by a factor of $\sim 2$. From the pulse height spectra measured by the HPGe detector it was found that the terrestrial gamma ray intensity at Mount Ho Huan was higher than that at Hsinchu campus only by $\sim 20 \%$.

\subsection{Fixed-point measurement}

We have also brought the real-time multichannel detector system on a bus to some representing and interesting places for fixed-point measurements of

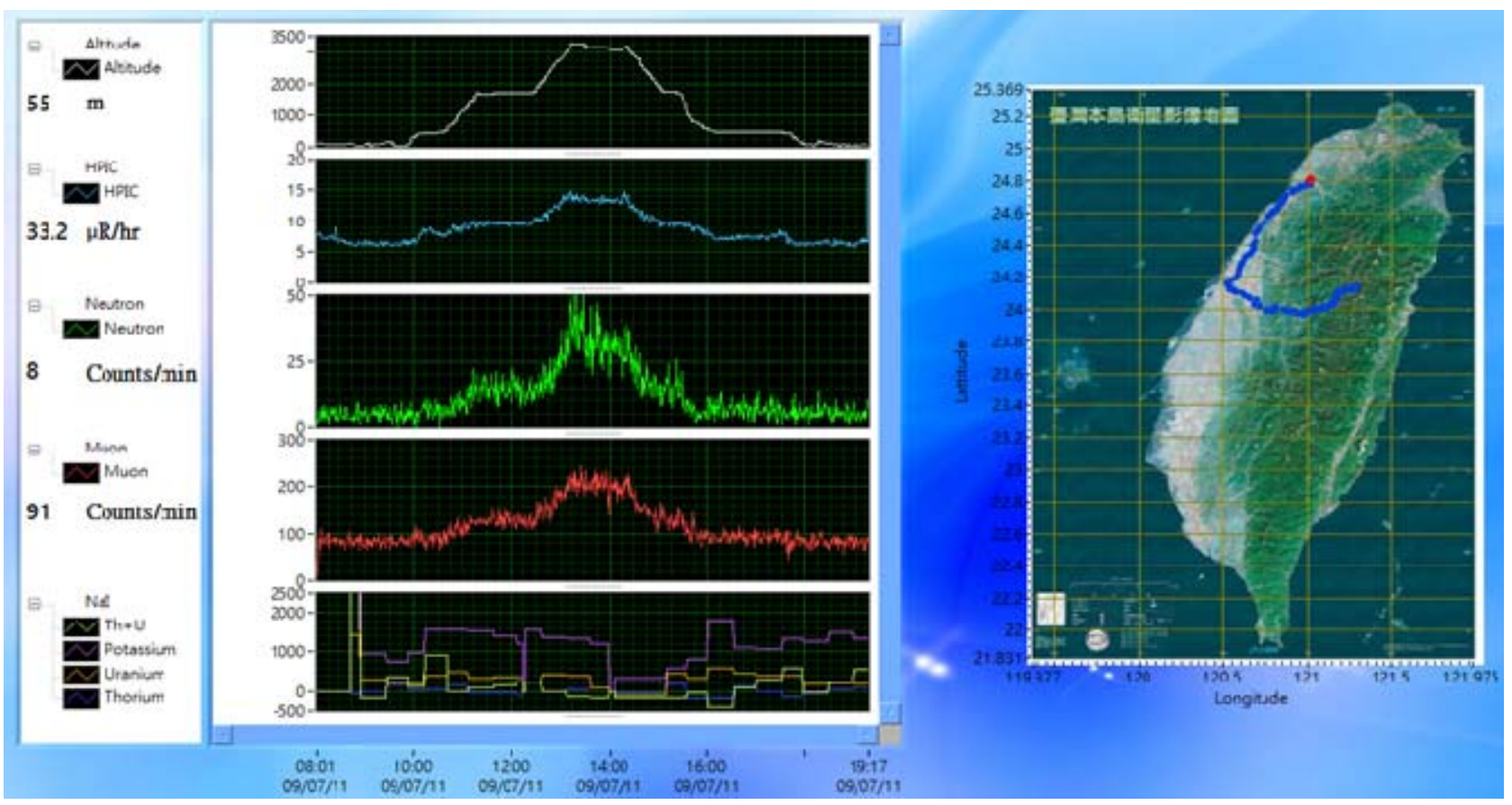

Figure 2. Real-time display of measured result from Hsinchu to Mount Ho Huan and return. 
Table 1. Measured result at different places.

\begin{tabular}{|c|c|c|c|c|c|c|}
\hline \multirow[t]{2}{*}{ Location } & \multicolumn{3}{|c|}{ HPGe(counts/hr) } & \multirow{2}{*}{$\begin{array}{l}\text { HPIC } \\
(\mu \mathrm{R} / \mathrm{hr})\end{array}$} & \multirow{2}{*}{$\begin{array}{l}\text { Neutron } \\
(\mathrm{cpm})\end{array}$} & \multirow{2}{*}{$\begin{array}{l}\text { Muon } \\
(\mathrm{cpm})\end{array}$} \\
\hline & U series & $\mathrm{K}-40$ & Th series & & & \\
\hline Tsing Hua campus indoor & 1918 & 5878 & 1562 & 10.4 & 2.6 & 71 \\
\hline Tsing Hua campus outdoor & 1357 & 4820 & 1204 & & & \\
\hline Mount Ho Huan & 1980 & 5844 & 1587 & $13.5^{*}$ & $32.7^{*}$ & $208^{*}$ \\
\hline Water surface Shyr-Men dam & $<\mathrm{MDL}$ & $<\mathrm{MDL}$ & $<\mathrm{MDL}$ & 4.3 & 2.5 & 83 \\
\hline Water surface Buh-Dai seaport & 20 & 135 & 19 & 4.0 & 2.7 & 76 \\
\hline Salt pile & 72 & 63 & 9 & 4.1 & NA & NA \\
\hline Buh-Dai seaport outdoor & 1494 & 5016 & 1002 & 9.2 & NA & NA \\
\hline Buh-Dai seaport inside bus & 384 & 1644 & 438 & 7.2 & 4.4 & 81 \\
\hline Coal pile & 408 & 927 & 228 & 5.3 & NA & NA \\
\hline Taichung Power Plant inside bus & 387 & 1962 & 477 & 6.8 & 5.2 & 78 \\
\hline
\end{tabular}

*Data measured inside a bus.

natural background radiation. Table 1 lists data of the measurements at Tsing Hua campus both indoor and outdoor, Mount Ho Huan, water surface in the middle of Shyr-Men dam, sea water surface of Buh-Dai seaport, the top of a salt pile neighboring Buh-Dai seaport, and the top of one of the coal piles at Taichung Power Plant. From Table 1 it is interesting to find following preliminary inferences: Fresh water contains essentially no natural radioactive nuclides, however, sea water contains a few percent compared with those in soil. Natural radioactive nuclides in salt pile are comparable with those in sea water, nevertheless, no significant content of ${ }^{40} \mathrm{~K}$ was found in salt pile as originally expected. Coal pile contains only $20-30 \%$ natural radioactive nuclides compared with those in soil. From the HPIC data for the measurements at the water surface both at Shyr-Men dam and Buh-Dai seaport, for which a few percent was assumed coming from skyshine gamma rays, the muon contribution was estimated to be $\sim 3.8$ $\mu \mathrm{R} / \mathrm{hr}$. The neutron counting rates at water surface were clearly lower than those on the ground, that is due to the air/water and air/ground interface effect. Comparing the measurements inside bus at Taichung Power Plant and Buh-Dai seaport with those outdoor in Buh-Dai seaport and Tsing Hua campus, the attenuation of the bus body to the terrestrial gamma rays from $U$ series $(609 \mathrm{keV})$, ${ }^{40} \mathrm{~K}(1.46 \mathrm{MeV})$, and $\mathrm{Th}$ series $(2.62 \mathrm{MeV})$ were found roughly to be a factor of $3.7,2.7$, and 2.4 , respectively, and that caused HPIC dose rate decreasing by $\sim 2.2$ $\mu \mathrm{R} / \mathrm{hr}$.

In addition to the fixed-point natural background radiation measurements, we also carried out a field measurement to Jinshan Nuclear Power Plant with two units of BWRs. When the bus past the turbine room building, we found that HPIC dose rate increased by a factor of around ten, ${ }^{3} \mathrm{He}$-detector counting rate increased by a factor of $\sim 12$ and the counting rate of the Berkeley Lab cosmic-ray detector increased by a factor of 2.6. The extraordinary high HPIC dose rate is obviously due to mainly the $6.13 \mathrm{MeV}$ gamma rays emitted from ${ }^{16} \mathrm{~N}$ decay. The high ${ }^{3} \mathrm{He}$-detector counting rate is clearly due to the neutrons emitted from the decay of ${ }^{17} \mathrm{~N}$, which is induced through ${ }^{17} \mathrm{O}(\mathrm{n}, \mathrm{p}){ }^{17} \mathrm{~N}$ reactions in the reactor core. The increase of counting rate of Berkeley Lab cosmic-ray detector is unexpected, it may be resulted from the high-energy electrons originating form skyshine of $6.13 \mathrm{MeV}$ gamma rays.

\section{Conclusion}

A real-time multichannel detector system consisting of a Berkeley Lab cosmic-ray detector, a moderated ${ }^{3} \mathrm{He}$ proportional counter, a HPIC, a $\mathrm{NaI}(\mathrm{Tl})$ spectrometer, and a HPGe spectrometer in couple with a GPS and with data acquired by a LabVIEW program in a notebook computer has been established to execute large-scale survey measurements of each component of natural background radiation. Large-scale natural background radiation measurements both on a train around the northern Taiwan coast and on a bus to Mount Ho Huan with an altitude of $\sim 3200 \mathrm{~m}$ above sea level have been successfully carried out to demonstrate the geophysical variation of the natural background radiation. Fix-point measurements in the campus, at Mount Ho Huan, on water surfaces of Shyr-Men dam and Buh-Dai seaport, at tops of salt pile and coal pile, and even in Jinshan Nuclear Power Plant have been carried out. From the measurement data, rough estimation of the contents of natural radioactive nuclides in fresh water, sea water, salt pile, and coal pile was made; attenuation of terrestrial gamma rays by the bus body was evaluated; and the hot radiation area near the turbine room of Jinshan Nuclear Power Plant has been identified.

\section{Acknowledgements}

The authors would like to express their sincere thanks to Dr. H. S. Matis, Lawrence Berkeley National laboratory for providing them three pieces of circuit board of the Berkeley Lab cosmic-ray detector. This work was funded by Natioanl Science Council, Taiwan, ROC under the contact number NSC 99-2221-E-007-076-MY3. 


\section{References}

[1] National Council on Radiation Protection and Measurement, Exposure of the Population in the United States and Canada from Natural Background Radiation, NCRP Report 94, (1987).

[2] UNSCEAR 2000 Report to the General Assembly, with Science Annexes, (2000).

[3] C. Twitty and H. Matis, Guide to Using the Berkeley Lab Cosmic Ray Detector, Lawrence Berkeley National Laboratory, LBNL-46909, (2002).

[4] M. Collier and L. Wolfley, Assembly Manual for the Berkeley Lab Cosmic Ray Detector, Lawrence Berkeley National Laboratory, LBNL-51419, (2006).

[5] J. W. Lin, Y. F. Chen, R. J. Sheu and S. H. Jiang, Measurement of angular distribution of cosmic-ray muon fluence rate, Nucl. Inst. and Meth. A619, (2010), pp. 24-27.

[6] R. J. Sheu and S. H. Jiang, Cosmic-ray induced neutron spectra and effective dose rates near air/ground and air/water interfaces in Taiwan. Health Phys. 84(1) (2003), pp. 92-99.

[7] L. Moens, J. De Donder, X. L. Lin, F. De Corte, A. De Wispelaere, A. Simonits and J. Hoste, Nucl. Inst. and Meth. 187 (1981), p. 451.

[8] S. H. Jiang, J. H. Liang, J. T. Chou, U. T. Lin and W. W. Yeh, A hybrid method for calculating absolute peak efficiency of germanium detectors. Nucl. Inst. and Meth. A413 (1998), pp. 281-292.

[9] Y. F. Chen, J. W. Lin, R. J. Sheu, U. T. Lin and S. H. Jiang, Measurement of natural background radiation intensity on a train. Rad. Prot. Dos. 144 (2011), pp. 663-66. 